\title{
Initial stage of GaN growth and its implication to defect formation in films
}

\author{
S. H. Cheung, L. X. Zheng, M. H. Xie, ${ }^{*}$ and S. Y. Tong \\ Department of Physics, The University of Hong Kong, Pokfulam Road, Hong Kong \\ N. Ohtani \\ Nippon Steel Corporation, Advanced Technology Research Laboratory, 5-10-1 Fuchinobe, Sagamihara, 229-8551 Japan
}

(Received 23 October 2000; revised manuscript received 8 March 2001; published 8 June 2001)

\begin{abstract}
In situ scanning tunneling microscopy (STM) observations of initial growth processes of GaN by molecularbeam epitaxy reveal important differences between growth on vicinal versus flat $\mathrm{SiC}(0001)$ substrates. Based on stop-growth STM studies, we explain why there are orders of magnitude reductions in the density of threading screw dislocations in the vicinal films. It is shown that on vicinal surfaces, three-dimensional (3D) islands develop into a characteristic shape. The islands coalesce much sooner than on flat surfaces. Consequently, fewer defects are created at their boundaries.
\end{abstract}

DOI: 10.1103/PhysRevB.64.033304

PACS number(s): 68.55.Jk, 68.37.Ef, 68.35.Bs, 68.35.Fx

In heteroepitaxy, one of the central concerns is the reduction of threading defects in epitaxial thin films as such defects are detrimental to devices made on such materials. ${ }^{1,2}$ This is particularly true for $\mathrm{GaN}$ growth, since most growth is carried out on substrates with large lattice and/or thermal mismatches with the nitride. ${ }^{3,4}$ In order to relieve the strain caused by such lattice and/or thermal mismatches, dislocations or other extended defects are generated at the interface between the epilayer and the substrate. If these defects are completely confined at the interface, they will not be very harmful to devices made on top of the layer that is far away from the interface. ${ }^{1}$ Unfortunately, defects such as dislocations generally thread up to the surface and thus traverse the entire thickness of the film. Efforts controlling dislocation dynamics thereby minimizing threading defect density are very relevant to the applications of the films.

For GaN, we have observed significant reduction of threading defects, especially threading screw dislocations by using vicinal substrates compared to that grown on flat ones. ${ }^{5}$ We shall show in this paper that such results are the consequences of characteristic differences in the nucleation and island coalescence processes. On vicinal surfaces, threedimensional (3D) islands take a characteristic shape showing the (0001) facets. Islands coalesce much sooner on the vicinal surface than on a flat one; therefore the island size is not fully developed for twisting. In addition, the underlying steps of the vicinal substrate act as an orientation guide preventing 3D islands from misalignment. Upon coalescence, fewer defects are created at their boundaries. This directly contrasts the case of flat films where relatively large and tilted 3D islands, upon coalescence, lead to creations of new defects at island boundaries.

Film growth and subsequent in situ surface examinations are conducted, respectively, in a molecular-beam epitaxy (MBE) chamber and a scanning tunneling microscopy (STM) chamber. The two facilities are interconnected with each other as well as with some other surface tools via ultrahigh vacuum (UHV) interlocks. The background pressures in all UHV chambers are in the range of $10^{-10}-10^{-11}$ mbar. In MBE, sources of atomic gallium $(\mathrm{Ga})$ and nitrogen $(\mathrm{N})$ are provided from a Knudsen cell and a radio-frequency plasma unit (Oxford Applied Research, CARS-25), with the latter being operated at $500 \mathrm{~W}$ and a $\mathrm{N}_{2}$ flow rate of $0.13 \mathrm{cc}$ per minute at STP. The Ga cell temperature is $980{ }^{\circ} \mathrm{C}$, giving rise to a $\mathrm{Ga} / \mathrm{N}$ flux ratio of 2 and a film growth rate of 0.1 bilayers/s (i.e., $0.26 \AA / \mathrm{A}){ }^{6}{ }^{6}$ The substrate temperature is $650^{\circ} \mathrm{C}$ throughout. Nominally flat and vicinal $\left(8^{\circ}\right.$ offcut towards $[11 \overline{2} 0]) 6 H-\mathrm{SiC}(0001)$ wafers are used as substrates. They are degreased in acetone and methanol before being loaded in vacuum. Deoxidization takes place in UHV at $1100^{\circ} \mathrm{C}$ under a $\mathrm{Si}$ flux. This procedure leads to atomically smooth surfaces and a $(\sqrt{3} \times \sqrt{3}) R 30^{\circ}$ surface reconstruction. For the vicinal substrate, characteristic terrace-step morphology

results. The step heights are measured to be mostly $7.6 \AA$ (i.e., $c / 2, c \approx 15.2 \AA$ is the lattice constant of $6 \mathrm{H}$-SiC), which agrees with that found during $\mathrm{SiC}$ epitaxial growth. ${ }^{7}$

In order to follow the initial nucleation processes of $\mathrm{GaN}$ on $\mathrm{SiC}$, specified amounts of materials are deposited in the MBE chamber followed by a rapid thermal quenching of the surface. Thermal quenching is achieved simply by switching off the alternating current flowing through the sample for substrate heating during deposition. The sample is then transferred to the adjacent STM chamber for surface morphology analyzes. Room temperature STM measurements are carried out under the constant current mode. The tunneling current is $0.1 \mathrm{nA}$ and sample bias is $-3.0 \mathrm{~V}$.

Figures 1(a)-1(f) show STM images, together with selected line profiles, of surfaces following $\mathrm{GaN}$ deposition on vicinal $\mathrm{SiC}(1000)$ for different nominal thicknesses. Clearly from the image of Fig. 1(a) the initial growth of $\mathrm{GaN}$ on $\mathrm{SiC}$ proceeds by $3 \mathrm{D}$ island formation. For the very early stage nucleation, as represented by the smallest islands in Fig. 1(a) the islands are spherical with rounded tops. As the islands become larger, they develop into a distinct shape showing a flat top, being the (0001) plane. The change from round-top islands to flat-top ones reflects the slower growth in the [0001] direction vs lateral directions. The flat-top feature can be clearly seen in the line profile of Fig. 1(a) which is obtained after firstly tilting the image by $8^{\circ}$ towards $[11 \overline{2} 0]$, so that the [0001] direction is horizontal. We have measured the height/lateral-size ratio of the islands and the results are sum- 

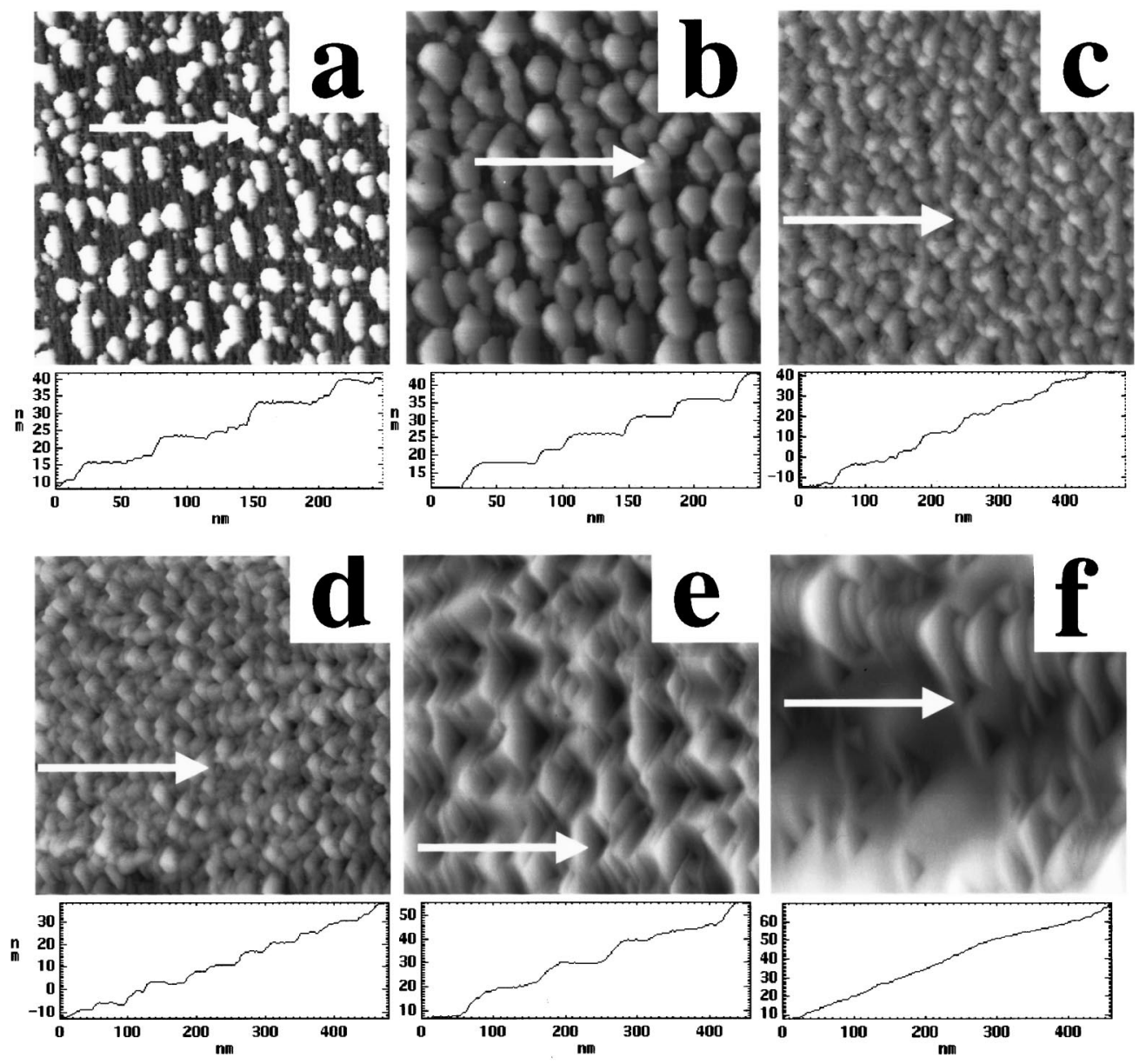

FIG. 1. STM images together with typical line profiles of surfaces following GaN deposition on a vicinal $\mathrm{SiC}(0001)$ substrate for film thicknesses of (a) $30 \AA$, (b) $80 \AA$, (c) $150 \AA$, (d) $250 \AA$, (e) $500 \AA$, and (f) $1500 \AA$. Image size: (a), (b): $500 \mathrm{~nm} \times 500 \mathrm{~nm}$; (c)-(f): $1000 \mathrm{~nm} \times 1000 \mathrm{~nm}$. The line profiles are obtained following an $8^{\circ}$ degree tilt of the image towards [11 $\left.\overline{2} 0\right]$, the direction of misorientation of the substrate.

marized in Fig. 2. It is seen that the ratio is a decreasing function of island size and its value converges to approximately $\tan 8^{\circ}=0.14$. Therefore, the larger islands become wedgelike in shape as illustrated by the line profile of Fig. 1(a). The island height $H$ and lateral dimension $L$ are related

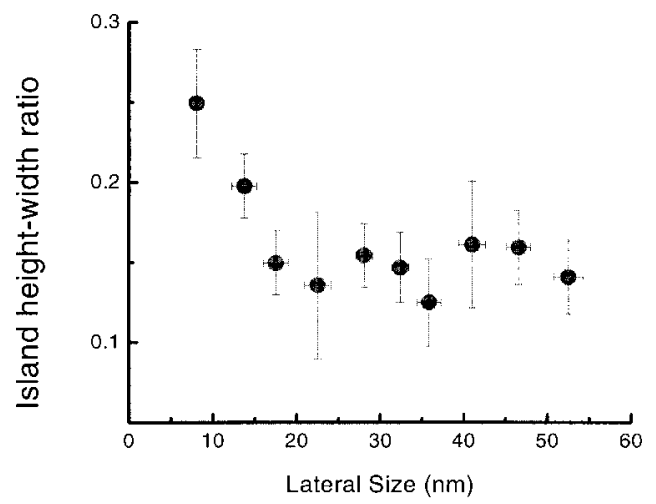

FIG. 2. Ratio between island height and lateral dimension (in the $[11 \overline{2} 0]$ direction) as a function of island size on vicinal substrates. by the vicinal angle of the substrate $\left(8^{\circ}\right)$ according to $H / L$ $=\tan 8^{\circ}$.

We first address the observation that on the vicinal substrate, the larger islands in Fig. 1(a) are elongated in the direction parallel to the step edge, i.e., [1 100$]$. This is related to the wedge shape of the islands. The rate of advance of an island without coalescence, in a given direction, depends on the height of the sidewall in that direction. Referring to Fig. 3(a), the sidewall $H$ in the [11 $\overline{2} 0]$ direction is higher than the average sidewall height $\left\langle H_{0}\right\rangle$ in the $[1 \overline{1} 00]$

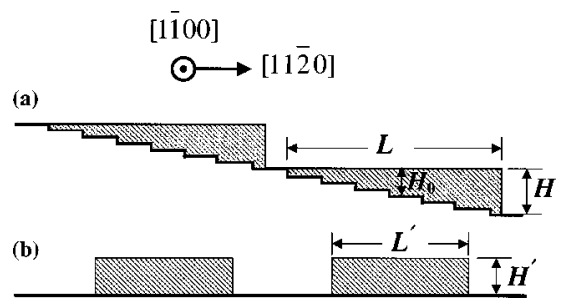

FIG. 3. Schematic diagram showing pre-coalesced $\mathrm{GaN}$ islands (a) when grown on vicinal and (b) flat $\mathrm{SiC}(0001)$ substrates. The volumes and island densities on the two substrates are the same. 

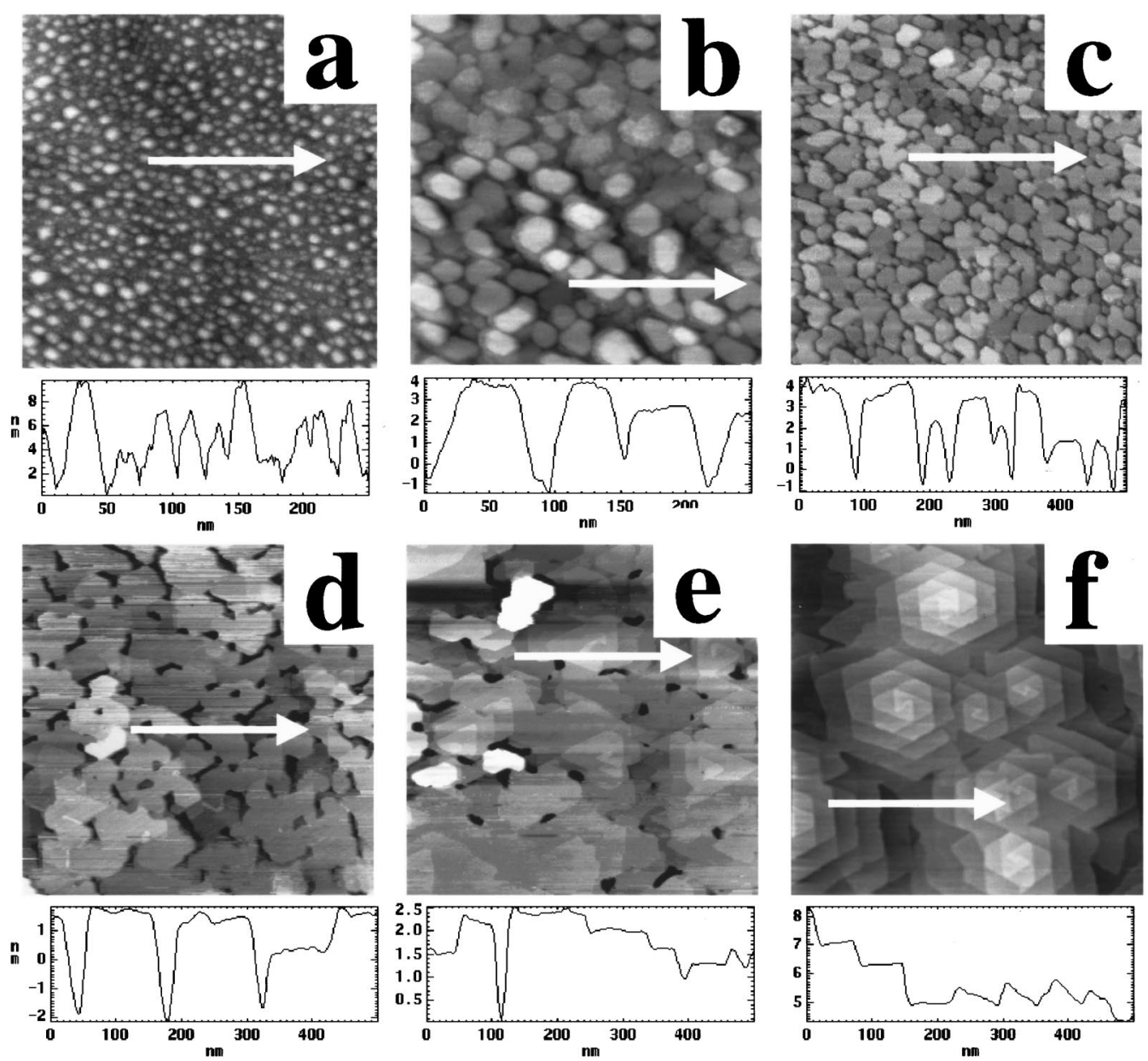

FIG. 4. STM images and line profiles of surfaces following GaN deposition on a nominally flat SiC(0001) substrate for film thicknesses of (a) $30 \AA$, (b) $80 \AA$, (c) $150 \AA$, (d) $250 \AA$, (e) $500 \AA$, and (f) $1500 \AA$. Image size: (a), (b): $500 \mathrm{~nm} \times 500 \mathrm{~nm}$; (c) $-(\mathrm{f}): 1000 \mathrm{~nm} \times 1000 \mathrm{~nm}$.

direction. Therefore, fewer atoms are needed to advance per unit distance along the [1 $1 \overline{1} 00]$ direction than along the $[11 \overline{2} 0]$ direction. As it is reasonable to assume that the same number of atoms arrive at each unit length of the sidewalls, the islands will grow into an elongated shape, with the larger dimension in the [1 100$]$ direction. Such laterally elongated islands are clearly seen in Figs. 1(a) and 1(b). The measured $L_{[1 \overline{1} 00]} / L_{[11 \overline{2} 0]}$ aspect ratio is approximately 1.5 . By comparison, the islands that nucleate on the flat substrate do not show any lateral elongation at all [see Figs. 4(a) and 4(b)].

As islands grow even bigger, they start to coalesce [see, e.g., Fig. 1(b)]. Due to the fact that these islands are elongated along $[1 \overline{1} 00]$, they coalesce first in this direction. Along [1120], coalescence comes later. Because the islands are multilayer high due to their 3D nature, coalescence in the $[11 \overline{2} 0]$ direction naturally leads to a step-bunched morphology [Figs. 1(c)-1(e)]. Comparing Fig. 1(e) with Figs. 1(c) and 1(d) there is also evident a transition of the step structure from a wavy one to a jagged morphology, showing segments of straight edges. This is the consequence of the presence of alternating type- $A$ and type- $B$ steps in a wurzite film. ${ }^{8}$ The straight edge segments are parallel to [2 $2 \overline{1} 0]$ or [ $\overline{1} 2 \overline{1} 0]$, belonging to the slow growing type- $B$ steps. Continued growth leads to a gradual debunching of the macrosteps into double bilayers $(\sim 5.2 \AA)$ (Ref. 8$)$ and thus a smoother surface morphology, as shown in Fig. 1(f). The driving force for debunching of macrosteps is the natural tendency for the film to minimize its surface and step energies as the film grows. From the panels of the figure, we estimate that complete coalescence of islands and the formation of a continuous film occurs at nominal thicknesses in the range $80-150 \AA$ [i.e., between Figs. 1(b) and 1(c)] on a vicinal substrate.

Figures 4(a)-4(f) show, for comparison, surfaces following $\mathrm{GaN}$ deposition on a nominally flat $\mathrm{SiC}(1000)$ for the same nominal thicknesses as those in Figs. 1(a)-1(f). Again from the images of Figs. 4(a) and 4(b), it is seen that initial $\mathrm{GaN}$ growth is by 3D island nucleation. However, unlike the situation of the vicinal film, the islands in Fig. 4 do not show asymmetry in lateral dimensions. Instead they are approximately circular or polygonal. The smallest islands are spherical in shape [Fig. 4(a)] while the larger islands develop flat tops of the (0001) plane [Fig. 4(b)]. The islands, before coalescence, exhibit a (lateral) length to height ratio of 3-5, agreeing with the findings of other works. ${ }^{9}$ In Fig. 4(b), some islands appear to have coalesced. From the panels of Fig. 4, we estimate that the formation of a continuous film on a flat substrate occurs at a thickness above $250 \AA$ [i.e., after the 
film of Fig. 3(d)]. This is much later than that on the vicinal substrate. As more islands coalesce, deep valleys and holes form at the boundaries between partially coalesced islands [Figs. 4(c) $-4(\mathrm{e})]$. The individual grains on different islands are likely to be randomly twisted due to strain. When coalescence is complete, spiral mounds emerge and subsequently become the dominant feature [Fig. 4(f)]. Twisting and tilting of different grains of the coalesced film are clearly evident in the line profile of Fig. 4(f) where the (0001) terraces belonging to different mounds are nonparallel. The angle of tilt is estimated to be more than $1^{\circ}$.

We turn to explain the reason why coalescence of islands occurs earlier on a vicinal substrate than on a flat substrate. Under identical deposition conditions, it is reasonable to assume that the nucleation rates and island number densities are similar on the two substrates. It follows then that the average volume of the individual islands before coalescence is the same on the two substrates. On a vicinal substrate, the height and lateral size of the wedge-shaped islands are related according to $H=L \tan 8^{\circ}$, so the volume of such an island is given by $V=(1 / 2) L^{2} \tan 8^{\circ}=0.07^{*} L^{2}$ (see Fig. 3). On a flat substrate, the islands are mesalike and the volume is given by $V=H^{\prime} L^{\prime}=L^{\prime 2} / \beta$, where $\beta=L^{\prime} / H^{\prime}$ is the length vs height ratio. By equating the two volumes, we find that $L / L^{\prime}=1 / \sqrt{0.07 \times \beta}$. As long as $L>L^{\prime}$, then the average lateral dimension of islands on a vicinal substrate is larger than that on a flat substrate. Because $L$ and $L^{\prime}$ are in the [11 $\left.\overline{2} 0\right]$ direction, it means that islands on a vicinal substrate will coalesce sooner in this direction than on a flat substrate. Furthermore, we have already shown that on the vicinal substrate, island coalescence in the [1 $\overline{1} 00]$ direction takes place even sooner than in the [ $11 \overline{2} 0]$ direction. Therefore, it would be generally true that island coalescence on a vicinal substrate occurs earlier than on a flat substrate, as long as the condition is $L>L^{\prime}$ satisfied, which means that $\beta$ must be less than $1 / 0.07=14$. Our data, presented in Fig. 4(a), as well as those of other works, ${ }^{9,10}$ show that $\beta$ is between 3 and 10 , supporting the fact that island coalescence occurs much earlier on vicinal substrates.

The earlier island coalescence on a vicinal substrate is responsible for the reduction of screw-type defects in the film. Screw dislocations can arise from the coalescence of islands if the lattices of different islands are significantly misaligned. ${ }^{11,12}$ On a flat surface, the islands are freestanding and relatively large before coalescence. As they have more time to grow, they are more likely to be misaligned and twisted at coalescence. Hence, a high density of defects appears in the film. On a vicinal substrate, on the other hand, the 3D islands are guided by the underlying steps to form wedge-shaped islands. Also, coalescence in the [1 $1 \overline{1} 00]$ direction occurs rather soon, followed by coalescence in the $[11 \overline{2} 0]$ direction. The earlier coalescence means that the islands are smaller and less likely to be twisted with respect to each other at coalescence.

The above discussions also explain the benefits of lowtemperature buffers commonly used in $\mathrm{GaN}$ epitaxy on heterogeneous substrates. ${ }^{13,14}$ Indeed, an effective way of achieving high-quality films is to nucleate a high density of islands and let them coalesce early. In this way, islands cannot develop fully in size and fewer defects are created at coalescence. Reducing the deposition temperature serves this purpose since low temperatures lead to high nucleation rates. Once coalescence is complete, further growth at raised temperatures is by homoepitaxial step flow and the film remains in good quality.

This work was supported by HK RGC Grant Nos. 7142/ 99P, 7121/00P, 9396/00P, N-HKU028/00, and U.S. Department of Energy Grant No. DE-FG-02-84ER45076.
*Corresponding author. Email address: mhxie@ @kusua.hku.hk

${ }^{1}$ R. Hull and J. C. Bean, in Strained-Layer Superlattices: Materials Science and Technology, Semiconductors and Semimetals, edited by T. P. Pearsall (Academic, New York, 1991), Ch. 1, Vol. 33.

${ }^{2}$ P. Kozodoy, J. P. Ibbetson, H. Marchand, P. T. Fini, S. Keller, J. S. Speck, S. P. DenBaars, and U. K. Mishra, Appl. Phys. Lett. 73, 975 (1998).

${ }^{3}$ Z. Sitar, M. J. Paisley, B. Yan, and R. F. Davis, Diamond Silicon, Carbide and Related Wide Bandgap Semiconductors, edited by J. T. Glass, R. F. Messier, and N. Fujimori, Mater. Res. Soc. Symp. Proc. No. 162 (Materials Research Society, Pittsburgh, 1990), p. 537.

${ }^{4}$ T. P. Humphreys, C. A. Sukow, R. J. Nemanich, J. B. Posthill, R. A. Rudder, S. V. Hattangady, and R. J. Markunas, Diamond, Silicon, Carbide and Related Wide Bandgap Semiconductors (Ref. 3), p. 531.

${ }^{5}$ M. H. Xie, L. X. Zheng, S. H. Cheung, Y. F. Ng, H. S. Wu, S. Y.
Tong, and N. Ohtani, Appl. Phys. Lett. 77, 1105 (2000).

${ }^{6}$ S. M. Seutter, M. H. Xie, W. K. Zhu, L. X. Zheng, H. S. Wu, and S. Y. Tong, Surf. Sci. Lett. 445, L71 (2000).

${ }^{7}$ T. Kimoto, A. Itoh, and H. Matsunami, Appl. Phys. Lett. 66, 3645 (1995).

${ }^{8}$ M. H. Xie, S. M. Seutter, W. K. Zhu, L. X. Zheng, H. S. Wu, and S. Y. Tong, Phys. Rev. Lett. 82, 2749 (1999).

${ }^{9}$ A. Pavlovska and E. Bauer, Surf. Rev. Lett. (to be published).

${ }^{10}$ Changwu Hu, David J. Smith, R. B. Doak, and I. S. T. Tsong, Surf. Rev. Lett. 7, 565 (2000).

${ }^{11}$ X. H. Wu, P. Fini, E. J. Tarsa, B. Heying, S. Keller, U. K. Mishra, S. P. DenBaars, and J. S. Speck, J. Cryst. Growth 189/190, 231 (1998).

${ }^{12}$ R. Lee Penn and J. F. Banfield, Science 281, 969 (1998).

${ }^{13}$ I. Akasaki, H. Amano, Y. Koide, K. Hiramatsu, and N. Sawaki, J. Cryst. Growth 98, 209 (1989).

${ }^{14}$ S. Nakamura, Jpn. J. Appl. Phys., Part 2 30, L1705 (1991). 